\title{
SPHENOSPORA KEVORKIANII, A RUST FUNGUS (UREDINALES: RAVENELIACEAE) ON THE ORCHID PLEUROTHALLIS MENTIGERA
}

\author{
Olinto L. Pereira; José R.P. Cavallazzi; Christtianno L. Rollemberg; Maria C.M. Kasuya* \\ Departamento de Microbiologia, Instituto de Biotecnologia Aplicada à Agropecuária/BIOAGRO, Universidade Federal de \\ Viçosa, Viçosa, MG, Brasil
}

Submitted: November 26, 2001; Returned to authors for corrections: April 08, 2002; Approved: June 10, 2002

\section{SHORT COMMUNICATION}

\begin{abstract}
A rust disease on the Brazilian orchid Pleurothallis mentigera was found in Mata do Jambreiro, a tropical forest in Minas Gerais State, caused by a fungus identified as Sphenospora kevorkianii Linder is described. $P$. mentigera Kraenzl. is added to $S$. kevorkianii host range.
\end{abstract}

Key words: Pleurothallis mentigera, Sphenospora kervokianii, Orchidaceae, rust, taxonomy.

Pleurothallis mentigera Kraenzl. is a rare epyphytical Brazilian orchid, found only in the tropical forest. During our survey of the mycobiota associated with Brazilian wild orchids, in June 2001, we found a rust disease on some leaves of $P$. mentigera (Tribe Epidendreae, Subtribe Pleurothallidinae) collected in Mata do Jambreiro, a protected area for biological conservation in Minas Gerais State, Brazil. The fungus was identified as Sphenospora kevorkianii Linder, which has been first reported from the orchid Epidendrum difforme Jacq. (Tribe Epidendreae, Subtribe Laeliinae) (3). The genus Sphenospora Dietel occurs in Africa, but primarily in the American tropics (3). In Brazil, S. kevorkianii has been reported on Cyrtopodium punctatum (L.) Lindl., Notylia lyrata F. Moore, Prescottia sclerophylla Lindley, Stanhopea graveolens Lindley and Zygostates lunata Lindley (2). According to the literature, this is the first report of occurence of a rust fungus on the Subtribe Pleurothallidinae (Orchidaceae).

Spermogonia and aecia unknown. Uredossorus hypophyllous, subepidermal, becoming erumpent. Uredospores ovoid, ellipsoid or subspherical, 28-33 x 18-26 $\mu \mathrm{m}$, membrane 1.5-3.5 $\mu \mathrm{m}$ thick, germ-pores obscure or one, equatorial. Telia subepidermal, becoming erumpent as an abundance of a dark orange colored oil (1), becoming dark-brown or black, $1 \mathrm{~mm}$

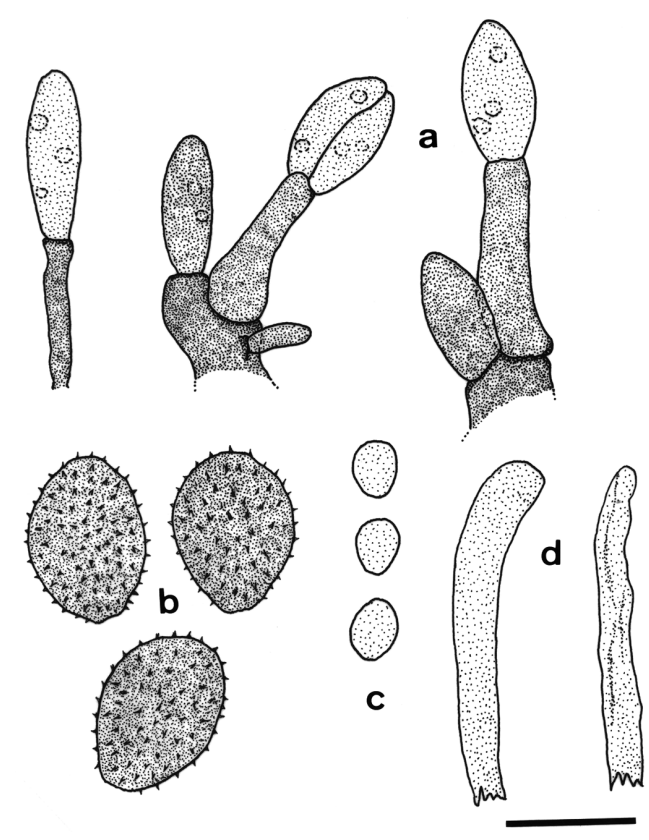

Figure1. Sphenospora kevorkianii. a. Teliospores. b. Uredospores. c. Basidiospores. d. Paraphyses. Scale bar: $25 \mu \mathrm{m}$.

\footnotetext{
* Corresponding author. Mailing address: Departamento de Microbiologia, Universidade Federal de Viçosa. 36571-000, Viçosa, MG, Brasil. Fax: (+5531) 899-2573, E-mail: mkasuya@ufv.br
} 
diam. Teliospores borne singly on pedicels, ellipsoid, thinwalled, 23-28 x 13-16 $\mu \mathrm{m}$. Paraphyses numerous, clavate or cylindrical, some curved, 100-116 x 8-10 $\mu \mathrm{m}$, yellow pigmented. Basidiospores yellowish, globular or irregularly ovoid, 8-11 x 6$7 \mu \mathrm{m}$.

Habitat: On leaves of Pleurothallis mentigera, Mata do Jambreiro, between Belo Horizonte and Nova Lima, Minas Gerais State, Brazil. Specimen examined: VIC 22171, deposited in VIC Herbarium, 19 november 2001.

\section{RESUMO}

\section{Sphenospora kevorkianii, fungo causador de ferrugem (Uredinales: Raveneliaceae) na orquídea Pleurothallis mentigera}

Uma nova doença causada por ferrugem foi encontrada em Pleurothallis mentigera, orquídea brasileira, na floresta tropical da Mata do Jambreiro, no Estado de Minas Gerais. O agente etiológico foi identificado como sendo Sphenospora kevorkianii. P. mentigera deve ser adicionada à lista de hospedeiros de S. kevorkianii.

Palavras-chave: Sphenospora kevorkianii, Pleurothallis mentigera, ferrugem, Orchidaceae, taxonomia.

\section{REFERENCES}

1. Cummins, G.B.; Hiratsuka, Y. Illustrated genera of rust fungi. The American Phytopathological Society. St. Paul, Minnesota, 1983, $152 \mathrm{p}$.

2. Hennen, J.F.; Hennen, M.M.; Figueiredo, M.B. Índice das ferrugens (Uredinales) do Brasil. Arquivos do Instituto Biológico. São Paulo, 1982, 201p.

3. Linder, D.H. A new rust of orchids. Mycologia, 36: 464-468, 1944. 